\title{
Antihypertensive Drugs (ACE- Inhibitors/ Beta- Blockers) and Chronic Infections as Potential Triggers for the Spontaneously Development of Small Plaque Parapsoriasis?!
}

\author{
Georgi Tchernev ${ }^{*}$, Uwe Wollina ${ }^{2}$, Konstantin Stavrov ${ }^{3}$ and Ivanka Temelkova ${ }^{1}$ \\ ${ }^{1}$ Medical Institute of Ministry of Interior (MVR), Department of Dermatology, Venereology and Dermatologic surgery, \\ General Skobelev 79, 1606. Onkoderma-Clinic for Dermatology, Venereology and Dermatologic Surgery, General Skobelev 26, 1606 Sofia. \\ ${ }^{2}$ Department of Dermatology and Allergology, Stadtisches Klinikum Dresden, Dresden, Germany. \\ ${ }^{3}$ Department of Dermatology, Venereology and Dermatologic surgery, General Skobelev 79, 1606 Sofia.
}

Received: July 17, 2019; Accepted: July 23, 2019; Published: July 26, 2019

*Corresponding author: Prof. Dr. Georgi Tchernev, Medical Institute of Ministry of Interior (MVR), Department of Dermatology, Venereology and Dermatologic surgery,General Skobelev 79, 1606. Onkoderma- Clinic for Dermatology, Venereology and Dermatologic Surgery, General Skobelev 26, 1606 Sofia.Email: georgi_tchernev@yahoo.de

\begin{abstract}
Background: There are different literature data according to which small plaque parapsoriasis (SPP) and guttate parapsoriasis could be considered as part of the various clinical forms of MF. However, there are no literature data that compare different control groups of patients (receiving/ not taking antihypertensive medication) and the two important possibilities deriving from it, namely: 1) that certain drug forms could be inducers of parapsoriasis and 2) that other drug forms could potentiate the transformation of existing parapsoriasis into T-cell lymphoma. We describe a case of possible infectious and / or drug-induced SPP by discussing an important, albeit currently hypothetical link to the drug mediated cancerogenesis.

Case report: We present a 39-year-old man with a disseminated eruptive erythemo-papulo-squamous rash, localized on the skin of the trunk and extremities. According to anamnestic data, skin symptoms date back to about 1 month when the patient was hospitalized in the ENT compartment for severe throat pain. Primary empirical antibiotic therapy with clindamycin $4 \times 600 \mathrm{mg} /$ daily i.v was performed on a regimen and partial remission was achieved. Immediately afterwards there was a resumption of symptoms and the additional occurrence of skin lesions. From the conducted tests, the presence of acute tonsillopharyngitis, focal infection of dental origin and elevated antistreptolysin titer was found. In parallel, the patient receives antihypertensive therapy (ACE inhibitor and beta blocker) on the occasion of arterial hypertension. There was a suspicion for infectious and / or drug-triggered psoriasis gutata, as the subsequent histological study showed evidence of small plaque parapsoriasis.

Conclusion: Although there are a number of literature data on the relationship between antihypertensive drugs and their pro-or anticancerogenic action against various types of tumors, there is currently no data available to compare the existing risk of developing T- cell lymphoma in patients with SPP and concomitant cardiac therapy with ACE inhibitors and beta blockers. We present a patient with triple histologically verified small plaque parapsoriasis and we are discussing a completely new pathogenetic element: triggering in the framework a possible chronic infection and systemic antihypertensive therapy. The selected retrospective or prospective analysis of wider groups of patients with chronic infections / systemic antihypertensive medication as well as proven $\mathrm{T}$ cell lymphomas could provide clarity with respect to the shared by us observations in single patients.
\end{abstract}

Keywords: Small plaque parapsoriasis; antihypertensive drugs; chronic infections; triggers; drug mediated cancerogenesis;

\section{Introduction}

At present, small plaque parapsoriasis (SPP) is considered as chronic idiopathic dermatosis [1]. There are no literature data, which examine the possibility that the disease may be medically or infrequently triggered. We describe the first case of parapsoriasis small plaque form, in which we determine the likelihood of induction by an active infectious process or drug intake.

\section{Case Report}

We present a 39-year-old man in good general condition and a complaint about rash on the skin of the trunk, upper and lower limbs. A month ago, the patient was hospitalized in the ENT department for severe throat pain. An empirical antibiotic therapy with clindamycin $4 \times 600 \mathrm{mg} /$ daily i.v was performed for 4 days with a temporary improvement, and after 4 days the throat pain resumed and in parallel there was occurrence of rash on the 
skin of the body. Re-treatment with levofloxacin $500 \mathrm{mg} \times 1$ / daily for 10 days according to the antibiogram was initiated, as a result of which the pains subsided, but the rash persisted. Ambulatory, two independent histological examinations were performed, according to which parapsoriasis data was established. The patient suffers from arterial hypertension for which he accepts perindopril/ indapamide $5 \mathrm{mg} / 1.25 \mathrm{mg}$ (1-0-0), nebivolol $5 \mathrm{mg}(1-0-0)$, as well as ademethionine (1-0-1) due to elevated levels of liver enzymes. Within the dermatological examination, a disseminated eruptive erythemo- papulo- squamous rash, localized on the skin of the trunk and extremities, was observed (Fig. 1a-1d). Based on the clinical data, there was a suspicion for infectious or drug-triggered psoriasis gutata. In the framework of additional studies, acute tonsillopharyngitis, dental infection (periodontitis chronica) and elevated values of antistreptolysin titer (AST)- $614.84 \mathrm{IU} / \mathrm{ml}$ were found. Due to the possibility, at the moment of hospitalization, that psoriasis guttata eruptiva (still without histological verification by our institution) could be medically triggered, a cardiologist was consulted, as a result of which the administration of perindopril / indapamide and nebivolol was discontinued and replaced with doxazosin $2 \mathrm{mg}$ (0- 0-1) and moxonidine $0.2 \mathrm{mg}$ (1-0-1). A skin biopsy was taken, and histological verification once again revealed the evidence for dilatated vessels and lymphocyte infiltrate with scarce dermatropy, and diagnosis of parapsoriasis with small plaques was made. Systemic therapy with methylprednisolone was performed according to a regimen ( 2 days $\times 32 \mathrm{mg} /$ per day intravenous, 2 days x $16 \mathrm{mg} /$ per day, followed by gradual dose reduction by $10 \%$ per week, per os), as well as chloropyramine hydrochloride x 1 amp. / i.m and esomeprazole x $40 \mathrm{mg} /$ daily, per os. An antibiotic treatment with ceftriaxone x $2 \mathrm{~g} /$ daily i.v for 7 days was performed on the occasion of the infectious process data and a significant improvement in the clinical picture was observed. Local therapy included calcipotriol / betamethasone gel- once a day. A mandatory eradication of the infectious focuses (acute tonsilopharyngitis and periodontitis chronicle) was recommended.
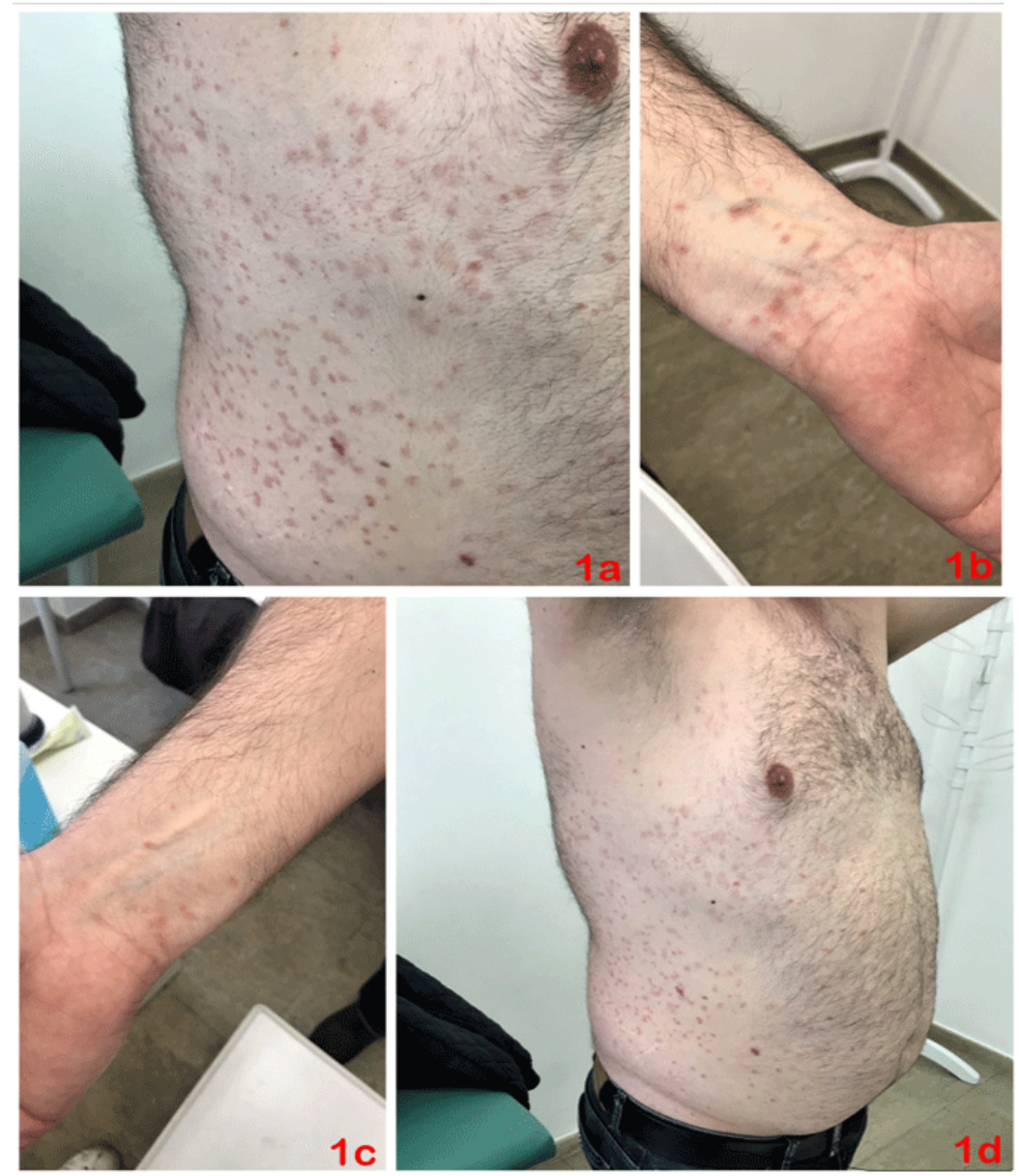

Figure. 1a- 1d: Clinical findig: the presence of disseminated eruptive erythemo- papulo- squamous rash, localized on the skin of the trunk and extremities 


\section{Discussion}

Due to the presence of a guttate clinical component in our case and a clinical picture corresponding to eruptive psoriasis guttate, we pose the question, whether if it is possible the triggers of psoriasis to be actually also inducers of parapsoriasis (with small plaques)? Certain authors even accept guttate parapsoriasis and digitate dermatosis (SPP) as variants of small plaque parapsoriasis [2]. How much these forms or subforms of parapsoriasis are provoked by different triggers-at the moment it is unclear!

Additionally, although defined as histopathologically nonspecific, SPP is considered to be predominantly a CD $4+\mathrm{T}$ cell-mediated disease that, in some cases, contains a dominant T-cell clone, which generates a doubt on the possibility of it being a direct precursor of cutaneous T-cell lymphomas [1].

According to certain authors' collectives, not only in large plaque, but also in small plaque parapsoriasis, progression to mycosis fungoides (MF) can be observed [3]. There are literature data suggesting that SPP and guttate parapsoriasis actually represent two of the many clinical faces of mycosis fungoides, and on the other hand, MF may be presented with psoriasiform plaques simulating psoriasis [4]. Indirectly, the latter is also supported by the hypothesis that the drug and infectious inducers of psoriasis induce parapsoriasis or at least the eruptive guttate form described in the present case.

Interestingly, there is currently no literature data on the possibility for development of mycosis fungoides in patients with small plaque parapsoriasis and systemic antihypertensive therapy (as well as those with or without chronic infections) versus those without antihypertensive medication. A number of literature data are linking antihypertensive medications with pro, as well as anticancerogenic anticancerogenic action with respect to various cancers. While beta-blockers have some protective action, according to certain authors in patients with histologically proven thick melanomas, the sartans (irbesartan and valsartan) have, according to the literature, pro-carcinogenic effects on the development of melanoma [5-8]. According to other authors, the intake of ACE inhibitors in turn is associated with an increased risk of developing carcinoma [9].
Literary data share the hypothesis of the probability of drug induced pseudolymphomas; including drug associated reversible $\mathrm{T}$ cell dyscrasias, which draw a strong morphological and phenotypic parallel with mycosis fungoides [5]. This also leads to the thought fot medication triggering of the small plaque form of parapsoriasis? And hence for a possible switch to MF, which also determines the need for regular clinical, paraclinical and histological controls even after the elimination of the possible trigger factor / component.

\section{References}

1. Haeffner A, Smoller B, Zepter K, Wood G. Differentiation and clonality of lesional lymphocytes in small plaque parapsoriasis. Arch Dermatol. 1995;131(3):321-4.

2. King Ismael D, Ackerman A. Guttate parapsoriasis/digitate dermatosis (small plaque parapsoriasis) is mycosis fungoides. Am J Dermatopathol. 1992;14(6):518-30.

3. Väkevä L, Sarna S, Vaalasti A, Pukkala E, Kariniemi AL, Ranki A. A retrospective study of the probability of the evolution of parapsoriasis en plaques into mycosis fungoides. Acta Derm Venereol. 2005;85(4):318-23. doi: 10.1080/00015550510030087

4. Azizpour A, Ghanadan A, Nasimi M, Etesami I. Psoriasiform mycosis fungoides: a rare form of the disease with review of the literature. Dermatol Online J. 2017.

5. Tchernev G, Temelkova I. Valsartan Induced Melanoma?! First Description in Medical Literature! Open Access Maced J Med Sci. 2018;6(12):2378-2380. doi: 10.3889/oamjms.2018.517

6. Tchernev G, Temelkova I. Drug-Induced Melanoma: Irbesartan Induced Cutaneous Melanoma! First Description in the World Literature! Open Access Maced J Med Sci. 2019;7(1):114-116. doi: 10.3889/ oamjms.2019.042

7. Tchernev G, Temelkova I. Irbesartan Induced Cutaneous Melanoma! Second Case in the Medical Literature!. Open Access Maced J Med Sci. 2019;7(1):121-123. doi: 10.3889/oamjms.2019.043

8. De Giorgi V, Grazzini M, Gandini S, Benemei S, Lotti T, Marchionni N et al. Treatment with $\beta$-blockers and reduced disease progression in patients with thick melanoma. Arch Intern Med. 2011;171(8):779-81. doi: 10.1001/archinternmed.2011.131.

9. Mueller P. Lung Cancer Risk with ACE Inhibitors./ Hicks M et al. Angiotensin converting enzyme inhibitors and risk of lung cancer: Population based cohort study. 2018;363:k4209. doi: 10.1136/bmj. $\mathrm{k} 4209$

10. Magro C, Daniels B, Crowson A. Drug induced pseudolymphoma. Semin Diagn Pathol. 2018;35(4):247-259. doi: 10.1053/j.semdp.2017.11.003 